\title{
THE $\lambda$-INTERSECTION BODIES AND AN ANALYTIC GENERALIZED BUSEMANN-PETTY PROBLEM
}

\author{
Denghui Wu, TONGYi MA AND LiLi ZHANG
}

Abstract. In this paper, we obtain an extension of connections between an analytic generalization of the Busemann-Petty problem and positive definite distributions. We show that the class of the $\lambda$-intersection bodies is closely related to the analytic generalized Busemann-Petty problem.

Mathematics subject classification (2010): 52A40, 52A20.

Keywords and phrases: Star body, $\lambda$-intersection body, generalized Busemann-Petty problem, generalized Minkowski-Funk transforms.

\section{REFERENCES}

[1] K. BALL, Some remarks on the geometry of convex sets, Geometric aspects of functional analysis (1986/87), Lecture Notes in Math. 1317, Spring-Verlag, Berlin-Heindelberg-New York, 1988, 224 231.

[2] J. Bourgain, On the Busemann-Petty problem for perturbations of the ball, Geom. Funct. Anal., 1, 1 (1991), 1-13.

[3] J. Bourgain, G. Zhang, On a generalization of the Busemann-Petty problem, Convex geometric analysis (Berkeley, CA 1996), Math. Sci. Res. Inst. Publ. 34, Cambridge Univ. Press, Cambridge, (1998), 65-76.

[4] H. Busemann, C. M. Petty, Problems on convex bodies, Math. Scand., 4, (1956), 88-94.

[5] R. J. GARDNER, Intersection bodies and the Busemann-Petty problem, Trans. Amer. Math. Soc., 342, 1 (1994), 435-445.

[6] R. J. GARDNER, A positive answer to the Busemann-Petty problem in three dimensions, Ann. Math., 140, 2 (1994), 435-447.

[7] R. J. Gardner, A Koldobsky and T. Schlumprecht, An analytic solution to the BusemannPetty problem on sections of convex bodies, Ann. Math., 149, 2 (1999), 691-703.

[8] A. Giannopoulos, A note on a problem of H. Busemann and C. M. Petty concerning sections of symmetric convex bodies, Mathematika, 37, (1990), 239-244.

[9] G. H. Hardy, J. E. Littlewood, G. Pólya, Inequalities, Cambridge Univ. Press, Cambridge, 1952.

[10] A. KoldobSKy, Intersection bodies in $\mathbb{R}^{4}$, Adv. Math., 136, 1 (1998), 1-14.

[11] A. Koldobsky, Fourier analysis in convex geometry, Mathematical Surveys and Monographs 116, Amer. Math. Soc., Providence, RI, 2005.

[12] A. KoldobSKy, A generalization of the Busemann-Petty problem on sections of convex bodies, Israel J. Math., 110, 1 (1999), 75-91.

[13] N. J. Kalton, A. Koldobsky, V. Yaskin And M. Yaskina, The geometry of $L_{0}$, Canada. J. Math., 59, 5 (2007), 1029-1040.

[14] D. G. LARMAn, C. A. Rogers, The existence of a centrally symmetric convex body with central sections that are unexpectedly small, Mathematika, 22, (1975), 164-175.

[15] E. LuTwaK, Intersection bodies and dual mixed volumes, Adv. Math., 71, 2 (1988), 232-261.

[16] M. PAPADImitraKis, On the Busemann-petty problem about convex, centrally symmetric convex bodies in $\mathbb{R}^{n}$, Mathematika, 39, (1992), 258-266.

[17] B. RUBIN, Intersection bodies and genaralized cosine transform, Adv. Math., 218, 3 (2008), 696-727. 
[18] B. Rubin, G. Zhang, Generalizations of the Busemann-Petty problem for sections of convex bodies, J. Funct. Anal., 213, 2 (2004), 473-501.

[19] G. Zhang, Intersection bodies and Busemann-Petty inequalities in $\mathbb{R}^{4}$, Ann. Math., 140, 2 (1994), 331-346.

[20] G. ZhAng, A positive solution to the Busemann-Petty problem in $\mathbb{R}^{4}$, Ann. Math., 149, 2 (1999), $535-543$.

[21] G. Zhang, Sections of convex bodies, Amer. J. Math., 118, 2 (1996), 319-340. 